\title{
Nutritional Adequacy of the Specific Carbohydrate Diet in Pediatric Inflammatory Bowel Disease
}

\author{
Kimberly Braly, RD¹, Nila Williamson, RD¹, Michele L. Shaffer, PhD ${ }^{1,2}$, Dale Lee, MD ${ }^{1}$, \\ Ghassan Wahbeh, MD¹, Jani Klein ${ }^{1}$, Matthew Giefer, MD¹, and David L. Suskind, MD1 \\ ${ }^{1}$ Department of Pediatrics, Division of Gastroenterology, Seattle Children's Hospital and \\ University of Washington (Seattle, Washington, U.S.A.) \\ ${ }^{2}$ Center for Clinical and Translational Research, Seattle Children's Research Institute (Seattle, \\ Washington, U.S.A.)
}

\begin{abstract}
Introduction-The Specific Carbohydrate Diet (SCD) is an exclusion diet used as a therapy in inflammatory bowel disease (IBD). The aim of this study was to evaluate the nutritional adequacy of the SCD.
\end{abstract}

\begin{abstract}
Methods-Prospective dietary data over 12 weeks were analyzed for pediatric patients on the SCD. Intake of 20 key nutrients was compared to Dietary Recommended Intake (DRI) levels and nutrient intake data from similarly aged children from The National Health and Nutrition Examination Survey (NHANES) National Youth Fitness Survey (NNYFS) in 2012.

Results-9 patients enrolled, with 8 patients completing the study. 6/8 individuals completing the study had gained weight, 1 individual had weight loss, and 1 had no change in weight. Energy intake was significantly greater than $100 \%$ of the Recommended Daily Allowance (RDA)/ Adequate Intake (AI) for $64 \%$ of daily intakes completed for this study. The majority of participants' daily intakes met or exceeded the RDA for vitamins B2, B3, B5, B6, B7, B12, C, A, and E. One hundred percent of participants' intakes were below the RDA for vitamin D. Seventyfive percent of daily intakes were less than the RDA for calcium. The upper limit was met or exceeded for magnesium in $42 \%$ of daily intakes. Average Vitamin A intake was significantly greater than the upper limit $(\mathrm{p}=0.01)$.
\end{abstract}

Discussion-Nutrient intake of pediatric IBD patients on the SCD was adequate when compared to a healthy peer reference population, but adequacy was variable when compared to the DRIs. Close monitoring with a multi-disciplinary team for patients using the SCD as an alternative or adjunct therapy is recommend to ensure positive outcomes for overall patient health.

\footnotetext{
Author for correspondence: David L. Suskind, M.D., Seattle Children's Hospital, 4800 Sandpoint Way NE, Seattle, Washington 98105, (Tel) 206-987-2521 (FAX) 206-987-2721, David.Suskind@ seattlechildrens.org.

Conflicts of Interest: None of the authors have a conflict of interest in regards to this article except for David Suskind who has written/ published Nutrition in Immune Balance (NIMBAL).

KB, NW, DL and DLS had substantial contributions to the conception or design of the work; KB, NW, DL, MS, GW, JK, MG and DLS contributed to the acquisition, analysis, or interpretation of data for the work; All authors were involved in drafting the work or revising it critically for important intellectual content; All all authors gave final approval of the version to be published; All authors gave agreement to be accountable for all aspects of the work in ensuring that questions related to the accuracy or integrity of any part of the work are appropriately investigated and resolved.
} 


\section{Keywords}

Inflammatory bowel disease; Crohn's disease; Ulcerative colitis; Specific Carbohydrate Diet; nutrition; Dietary Recommend intake (DRI)

\section{Introduction}

Complementary and alternative therapies are frequently utilized by patients with inflammatory bowel disease (IBD) with changes in dietary intake being commonplace. While the number of individuals on dietary therapy is unknown, 36-50\% of pediatric patients with IBD utilize some form of alternative therapy. ${ }^{1}$ In addition, over $75 \%$ of IBD patients restrict food groups based on subjective worsening of symptoms. ${ }^{2}$ The Specific Carbohydrate Diet (SCD), an exclusionary diet, has been utilized by many patients as an alternative or adjunct therapy. This diet first developed in the 1930s to treat celiac disease and then popularized in the 1990s, has emerged as a potential diet therapy for patients with IBD. ${ }^{3,4}$ Case series and reports from our institution and others have demonstrated not only clinical improvements and remission but normalization of inflammatory markers for patients with IBD.. ${ }^{5-7}$ While the mechanism of action for dietary therapy is not known, attention has focused on the ability of diet to alter the fecal microbiome as well as the effect of diet on immune barrier function. ${ }^{8}$

While nutritional therapy in the form of exclusive enteral nutrition (EEN) has a well established role in treatment of active Crohn's disease in pediatric patients, the SCD has primarily been utilized outside of the medical establishment. Patients have exhibited great interest in this dietary approach to IBD therapy, but often times this is without guidance from the medical team. ${ }^{9}$ The SCD excludes all grains, dairy products except for fully fermented yogurts and some hard cheeses and sweeteners except for honey. In addition, food additives such as emulsifiers, found in many processed foods, are excluded. Staples within the diet include meats, nut flours, fruits, legumes and vegetables. While EEN has been shown to meet nutritional requirements of patients with IBD, the dietary adequacy of the SCD has not been evaluated.

With evidence of clinical efficacy for the SCD as well as increasing patient interest in the diet, the nutritional adequacy and any potential nutritional risks of the diet need to be evaluated. Malnutrition in IBD is associated with increased morbidity, and restrictive diets have the potential to increase this risk by contributing to micronutrient deficiencies and/or inability to meet energy and protein needs. Concern has been raised with restrictive diets in IBD, such as the SCD, for the potential to induce deficiency in folate, thiamin, vitamin B6, calcium, potassium, and vitamins $\mathrm{A}, \mathrm{C}$, and $\mathrm{D} .^{2}$ To evaluate the nutritional adequacy of the SCD, we compared nutrient intake of pediatric patients following the SCD to Dietary Reference Intake (DRI) levels and population based intake for 20 key nutrients. 


\section{Methods}

\section{Study Design}

This is a single center analysis of nutrient intake in an open-label study designed to determine nutrition adequacy of the specific carbohydrate diet in pediatric patients with inflammatory bowel disease. The protocol was approved by the Institutional Review Board of Seattle Children's Hospital. All patients/participants provided written informed consent or assent. The study was registered with ClinicalTrials.gov (number: NCT02213835). Study participants were recruited from Seattle Children's Hospital from April 2014 to August 2015.

\section{Participants}

Patients with $\mathrm{CD}$ or UC with mild or moderate disease activity as defined by Pediatric Crohn's disease activity index (PCDAI) score of 10-45 or Pediatric Ulcerative colitis index (PUCAI) of 10-60 were enrolled into this study. The maximum PCDAI in this study was 45, and the maximum PUCAI was 55. Prior to the study no change in medication(s) for IBD could occur for a minimum of one month for immunosuppressive medications and two months for biologics.

\section{Study Intervention}

Patients went on to the SCD as the sole intervention for the study. Patients received one-onone education and counseling by a dietitian trained in the SCD during each visit. Prior to each visit patients completed a 3-day food intake record to help assure compliance and adequate intake while on the diet. Dietary guidance included overview of the SCD; which foods are included and excluded. The dietitian counseled on weight loss prevention/ management and provided several resources to help with meal planning such as recipe books and meal and snack recommendations. A staged approach which involved introduction of new SCD foods in a stepwise manner, working towards the complete SCD was utilized. Patients followed up and were in contact with the dietitian, research assistant and primary gastroenterologist for questions and troubleshooting over the 12 -week study. ${ }^{10}$ Patients had clinical follow-up at 2 weeks, 4 weeks, 8 weeks and 12 weeks. Dietary assessment included a 3-day detailed food intake record with date, time, food/drink, portion, description of how the food was prepared, amount eaten, and recipe when indicated. These food intake records were provided by each family at every follow-up visit. Counseling sessions involved weight loss prevention, increasing efficiency with meal planning, cooking, and diet variety. Based on diet recalls, some individuals were recommended to either start a multivitamin with mineral supplement and/or vitamin D supplement. These supplements were not included in nutrient analysis summary due to variable compliance.

\section{Diet Analysis}

Diet analysis of all detailed food intake records was completed using The Food Processor version 10.12.0 (ESHA Research, Salem, Oregon, USA) for each study participant. To ensure accuracy of nutrient content, recipes were built within the program for all homemade foods and baked goods. Intake of 20 key nutrients was then compared to DRI levels and 
nutrient intake data from The National Health and Nutrition Examination Survey (NHANES) National Youth Fitness Survey (NNYFS $)^{11}$. NNYFS collected nutrient information from healthy children in 2012 using in-person dietary interview to capture 24hour recall data. The full list is shown in Table 1.

\section{Other Measures}

Vitamin D 25-OH levels were assessed at baseline and upon completion of the 12-week intervention.

Patients with low vitamin D 25-OH levels $<30 \mathrm{ng} / \mathrm{mL}$ at baseline were supplemented with an SCD approved D supplement.

\section{Statistical Analysis}

Descriptive summaries were prepared for all variables including mean, standard deviation, quartiles, and range for quantitative variables (e.g., age, nutrient intakes) and frequencies and percentages for categorical variables (e.g., gender, receipt of supplementation). After preliminary visualization of daily intake values, nutrients that were markedly skewed right were log-transformed for analyses. All dietary data for patients were included as separate intakes in analyses, representing up to 12 daily intakes per patient. Comparisons to recommendations were based on the difference between the daily value and the RDA, AI or the upper limit (UL). Linear mixed-effects models ${ }^{12}$, a generalization of one-sample t-tests in this setting, were used to account for the multiple daily available per patient. For comparison to NNYFS nutrient intake data, NNYFS data were first subset to include only participants in the age range of the SCD patients. Next, NNYFS data were summarized to estimate population means. SCD patient data were compared to these population means utilizing one-sample t-tests. P-values less than 0.05 were considered statistically significant. All statistical analyses were conducted using SAS Version 9.4 (SAS Institute Inc., Cary, NC, USA) and R Version 3.0.3. ${ }^{13}$

\section{Results}

\section{Subjects}

Nine individuals from Seattle Children's Hospital enrolled in the study. Baseline demographics and clinical characteristics are shown in Table 2. Mean age of participants was $13.6 \pm 2.0$ years (range $11-17$ years). Average disease duration prior to study was 1.6 \pm 1.7 years (range $0-5$ years). One patient stopped the study at 2 weeks because of difficulty maintaining the diet. By week twelve, 7 out of 8 patients achieved clinical remission based on PCDAI/PUCAI scoring, normalization of labs, and clinical assessment. Diet therapy was ineffective for one patient. ${ }^{10}$

\section{Growth: Weight/Height Velocities}

Anthropometric measurements of weight and height over the 3 month trial varied significantly for patients within the study. While the $6 / 8$ individuals completing the study had positive weight gain, 1 individual had weight loss, and 1 had no change. The mean weight $\mathrm{z}$-score for participants who completed the study was $-0.29(\mathrm{SD}=0.73)$ with a mean 
increase over the course of the study of $0.14(\mathrm{SD}=0.28)$. The mean height $\mathrm{z}$-score for participants who completed the study was $-0.19(\mathrm{SD}=0.88)$ with a mean increase over the course of the study of 0.12 ( $\mathrm{SD}=0.14$ ). (Figure 1). BMI $\mathrm{z}$ score for all individuals that completed the study remained greater than -1 .

\section{Diet Analysis}

Energy intake was significantly greater than $100 \%$ of the RDA/ AI for $64 \%$ of daily intakes completed for this study. The mean energy intake ranged from $88-145 \%$ of the RDA. $2 / 8$ study participants had mean energy intake $<97 \%$ of the RDA. Similarly, protein intake was significantly higher than the NHANES reference population at approximately three times the RDA for protein for all individuals' average daily intakes (Figure 2).

The majority of participants' daily intakes met or exceeded the RDA for vitamins B2 (82\%), B3 (67\%), B5 (70\%), B6 (90\%), B7 (74\%), B12 (82\%), C (76\%), A (92\%), E (55\%). Seventy-one percent of participants' daily intakes were below the RDA for vitamins B1 and sixty-seven percent for B9. One-hundred percent of participants ' daily intakes were below the RDA for vitamin D. Fifty-six percent of participants' daily intakes met the RDA for vitamin K (Figure 3). Seventy-four percent of participants' daily intakes exceeded the RDA for iron, and $91 \%$ for selenium (Figure 4). Forty-eight percent of intakes met RDA for zinc and forty-two percent for magnesium. Forty-six percent on average met the RDA for phosphorus. Seventy-five percent of daily intakes were less than the RDA for calcium.

The upper limit was met or exceeded for magnesium in $42 \%$ of participants on average and in $66 \%$ of participants on average for vitamin A (Figure 5). The average Vitamin A intake was significantly greater than the upper limit $(\mathrm{p}=0.01)$.

\section{Other Measures}

Patients 2, 4, and 7 presented with hypo vitaminosis D with level $<30 \mathrm{ng} / \mathrm{mL}$ at our institution. Out of these patients, all started on varying dosages of SCD approved vitamin D. Patient 2 began vitamin D3 supplementation at 200 IU/day with improvement in levels from $18 \mathrm{ng} / \mathrm{ml}$ to $25 \mathrm{ng} / \mathrm{mL}$. Patient 4 presented with a level of $26 \mathrm{ng} / \mathrm{ml}$, started on $2000 \mathrm{IU} /$ day D3 with follow up level of $28 \mathrm{ng} / \mathrm{mL}$. This patient reported taking the supplement irregularly. Patient 7 started the study with a level of $17 \mathrm{ng} / \mathrm{mL}$, initiated $2000 \mathrm{IU} / \mathrm{day}$ vitamin D3, and completed the study with a level of $36 \mathrm{ng} / \mathrm{mL}$. Other participants either presented on vitamin D supplementation already, or did not require supplementation during the study.

\section{Discussion}

With recent reports suggesting efficacy of the SCD in patients with IBD, analysis of nutrient intake is essential to assure the nutritional adequacy of the SCD for these patients. The results of this study show that in a small cohort of pediatric patients with active IBD, intake on the SCD was comparable to that of healthy peers in the NNYFS for 20 key nutrients.

The DRIs provide reference values for energy and nutrient intake in healthy individuals. They are used for assessing diets for individuals and groups, setting goals to be achieved 
over time. These were established as guidelines for adequate nutrition in hopes of reducing the incidence of undernutrition and micronutrient deficiencies. The AI is used when data are insufficient to establish an RDA and is set at a level assumed to ensure nutritional adequacy. The disadvantage with utilizing DRIs in relation to this study include the lack of reference values for individual with chronic disease or malabsorptive states. This may underestimate the true needs of our patient population. In addition there is not an RDA established for many key nutrients, and some of these reference values are based on supplementation and not dietary intake. We do not currently have a gold standard for nutrient adequacy in pediatric IBD. Therefore, the DRIs and NNYFS were used as comparison.

The mean energy intake of our study participants varied from $88-144 \%$ of the RDA. $2 / 9$ study participants had energy intake $<97 \%$ of the RDA. A mean energy intake less than $80 \%$ of the RDA has been associated with reduced growth velocity. ${ }^{14}$ In a previous study on dietary intake of children with Crohn's Disease, $47 \%$ consumed $<80 \%$ of the RDA, a much higher percentage than what we observed in our cohort despite additional dietary limitations. ${ }^{15}$ Other studies comparing energy intake to calculated estimated energy requirements (EER) or intake of healthy controls have also found intake of children with active disease to be inadequate. ${ }^{16,17} \mathrm{Six}$ of eight patients that completed the study in our cohort had weight gain demonstrating that despite the exclusionary nature of the SCD, the majority of patients remained able to consume adequate energy intake to support growth despite the increased energy needs that we know are associated with active disease. Protein intake was three times the RDA indicating sufficient protein and amino acid intake.

In our analysis of the $\mathrm{SCD}$, mean intake of vitamins B1 (thiamin), B9 (folate), D, calcium, and phosphorus were found to be less than $100 \%$ of the RDA/AI reference values. A percentage of patients met the RDA for the remaining B vitamins, as well as zinc and magnesium intake, while others did not meet the RDA for these nutrients (see table 1). While not statistically significant, the average trajectory of nutrient intake relative to RDA/AI for the SCD patients showed a trend toward increasing intake for all nutrients with the exception of B3, zinc, and selenium. (Figure 6. Supplemental material) Given the exclusion of fortified grains and the limited dairy products of the diet the lower intake of these nutrients is understandable. The majority of our patients were not on an SCD multivitamin at the time of this study, however, given these results SCD multivitamins are now recommended when nutritional assessments suggest lower intake of micronutrients. Despite intake being lower than reference values, we were reassured when comparison to NHANES data demonstrated no significant differences in nutrient intake between pediatric patients on the SCD and population data from healthy peers of the same age and gender. Vitamin D was the only nutrient in our analysis which was significantly less than the RDA. However as discussed above, the low intake of individuals within our cohort was similar to that of data from healthy peers. In our population this may be been partially due to the avoidance of foods that are conventionally fortified. Due to malabsorption and increased intestinal losses of nutrients associated with the inflammation of IBD, it is possible that even if intake of Vitamin D was similar to the RDA it may not support normal serum levels. Vitamin D deficiency is common in children and adolescents with IBD, affecting up to $34.6 \%$ of patients. ${ }^{18}$ Vitamin D plays a role in the immune system of the gut and is emerging as a nutrient that may play a role in regulating disease severity in IBD. ${ }^{19}$ As such, 
individuals with IBD, including those following the SCD, should have monitoring of serum levels and supplementation as needed to prevent and treat deficiency.

The mean calcium intake of our study participants varied from 58-144\% of the RDA of 1300 $\mathrm{mg}$ /day. Given that dairy products on the SCD are limited to homemade yogurt and some hard cheeses, it is understandable that intake could be lower than recommendations. However, other studies of pediatric IBD patients without dietary restrictions also report inadequate calcium intake. In a recent study of 68 pediatric IBD patients, half of study participants consumed $<50 \%$ of the RDA for calcium. Dairy products are a frequently selfimposed diet restriction with IBD patients.${ }^{16}$ Multiple other studies have found calcium intake of children with IBD to be significantly less than the RDA and/or intake of healthy controls ${ }^{17,20,21}$. Poor dietary intake of calcium has the potential to impact bone health and increase risk of bone disease and therefore should be evaluated in IBD patients by a healthcare practitioner or dietitian.

The only nutrient found to exceed the upper limit within our study for the majority of individuals was Vitamin A. In spite of this, intake of Vitamin A was not significantly different than data from NHANES. It is hypothesized to be related to the high intake of eggs and vitamin A rich produce within the diet. None of the study participants demonstrated signs or symptoms of toxicity and after the study was completed, serum Vitamin A levels checked for a few individuals were found to be normal. Additionally, the upper limit for magnesium was met or exceeded in $50 \%$ of participants. Of note, the UL for magnesium was established based on a pharmaceutical supplement which could pose varying bioavailability to that of whole-food rich magnesium. Magnesium toxicity can result in diarrhea, and the majority of individuals experienced improvement in stool consistency aside from one participant that suffered from constipation.

This study also highlights the importance of a multidisciplinary approach to the treatment of IBD especially when incorporating dietary therapy. With patients and parents having variable nutrition knowledge it is essential to have both physician and dietitian working in concert addressing not only disease activity but also nutritional intake and status. Within both our research and clinical dietary program at Seattle Children's Hospital, we spend time addressing all patient questions regarding the diet and highlight nutritional concerns proactively with appropriate nutrient supplementation when indicated.

In conclusion, this study demonstrates that on the SCD, nutrient intake is comparable to that of similarly aged healthy children in the US. An SCD multivitamin with mineral and/or vitamin D supplement may be indicated depending on diet variety, as individuals with inadequate intake of allowed foods on the SCD may be lacking as seen with the nutrient intake of some of our patients as compared to the DRIs. Patient access to a dietitian and multi-disciplinary team with knowledge on the SCD likely had a positive influence on the overall nutritional quality of the diets for the children and adolescents in this study and is highly recommended to ensure the best outcomes for patients. While controlled trials are needed to determine the efficacy of the SCD in treating and controlling disease activity in patients with IBD, our data show nutritional adequacy and safety of the diet in general. The sample size of this study is small, and we are currently designing controlled follow-up 
studies to increase our knowledge of the dietary intervention. Future studies should evaluate whether similar results are found in adult populations on the SCD. Additionally, future studies need to assess whether gradual liberalization of the SCD can make it easier for patients to consume a nutritionally adequate diet while maintaining similar outcomes in the treatment of their IBD.

\section{Supplementary Material}

Refer to Web version on PubMed Central for supplementary material.

\section{Acknowledgments}

Source of Funding: This work was supported by grants from the Keating Foundation, and Seattle Children's Center for Clinical and Translational Research Academic Enrichment Fund. This publication was also supported by the National Center for Advancing Translational Sciences of the National Institutes of Health under Award Number UL1TR000423. The content is solely the responsibility of the authors and does not necessarily represent the official views of the National Institutes of Health.

\section{References}

1. Wong AP, Clark AL, Garnett EA, et al. Use of complementary medicine in pediatric patients with inflammatory bowel disease: results from a multicenter survey. Journal of pediatric gastroenterology and nutrition. 2009; 48(1):55-60. [PubMed: 19172124]

2. Hwang C, Ross V, Mahadevan U. Popular exclusionary diets for inflammatory bowel disease: the search for a dietary culprit. Inflammatory bowel diseases. 2014; 20(4):732-741. [PubMed: 24562173]

3. Gottschall, E., editor. Breaking the Viscious Cycle. 2nd ed. Kirkton Press Limited; 1994.

4. Haas SV, Haas MP. The treatment of celiac disease with the specific carbohydrate diet; report on 191 additional cases. The American journal of gastroenterology. 1955; 23(4):344-360. [PubMed: 14361377]

5. Suskind DL, Wahbeh G, Gregory N, Vendettuoli H, Christie D. Nutritional therapy in pediatric Crohn disease: the specific carbohydrate diet. Journal of pediatric gastroenterology and nutrition. 2014; 58(1):87-91. [PubMed: 24048168]

6. Obih C, Wahbeh G, Lee D, et al. Specific carbohydrate diet for pediatric inflammatory bowel disease in clinical practice within an academic IBD center. Nutrition. 2016; 32(4):418-425. [PubMed: 26655069]

7. Cohen SA, Gold BD, Oliva S, et al. Clinical and mucosal improvement with specific carbohydrate diet in pediatric Crohn disease. Journal of pediatric gastroenterology and nutrition. 2014; 59(4): 516-521. [PubMed: 24897165]

8. Vindigni, Stephen, Z, T., Suskind, David L., Damman, Christopher. The intestinal microbiome, barrier function, and immune system in inflammatory bowel disease: a tripartite pathophysiological circuit with implications for new therapeutic directions. Ther Adv Gastroenterol. 2016:1-20.

9. Suskind DL, Wahbeh G, Cohen SA, et al. Patients Perceive Clinical Benefit with the Specific Carbohydrate Diet for Inflammatory Bowel Disease. Digestive diseases and sciences. 2016; 61(11): 3255-3260. [PubMed: 27638834]

10. Suskind DL, Cohen SA, Brittnacher MJ, et al. Clinical and Fecal Microbial Changes With Diet Therapy in Active Inflammatory Bowel Disease. J Clin Gastroenterol. 2016

11. Borrud L, Chiappa MM, Burt VL, et al. National Health and Nutrition Examination Survey: national youth fitness survey plan, operations, and analysis, 2012. Vital Health Stat 2. 2014; (163): $1-24$.

12. Laird NM, Ware JH. Random-effects models for longitudinal data. Biometrics. 1982; 38(4):963974. [PubMed: 7168798] 
13. R: A language and environment for statistical computing. R Foundation for Statistical Computing. 2014. http://www.R-project.org/

14. Betts PR, Magrath G. Growth pattern and dietary intake of children with chronic renal insufficiency. Br Med J. 1974; 2(5912):189-193. [PubMed: 4364943]

15. Thomas AG, Taylor F, Miller V. Dietary intake and nutritional treatment in childhood Crohn's disease. Journal of pediatric gastroenterology and nutrition. 1993; 17(1):75-81. [PubMed: 8350215]

16. Hartman C, Marderfeld L, Davidson K, et al. Food Intake Adequacy in Children and Adolescents with Inflammatory Bowel Disease. Journal of pediatric gastroenterology and nutrition. 2016

17. Pons R, Whitten KE, Woodhead H, Leach ST, Lemberg DA, Day AS. Dietary intakes of children with Crohn's disease. Br J Nutr. 2009; 102(7):1052-1057. [PubMed: 19402936]

18. Pappa HM, Grand RJ, Gordon CM. Report on the vitamin D status of adult and pediatric patients with inflammatory bowel disease and its significance for bone health and disease. Inflammatory bowel diseases. 2006; 12(12):1162-1174. [PubMed: 17119391]

19. Verlinden L, Leyssens C, Beullens I, et al. The vitamin D analog TX527 ameliorates disease symptoms in a chemically induced model of inflammatory bowel disease. J Steroid Biochem Mol Biol. 2013; 136:107-111. [PubMed: 23000190]

20. Hendricks KM, Williams E, Stoker TW, Schoenfeld DA, Walker WA, Kleinman RE. Dietary intake of adolescents with Crohn's disease. J Am Diet Assoc. 1994; 94(4):441-444. [PubMed: 8144814]

21. Vernia P, Loizos P, Di Giuseppantonio I, Amore B, Chiappini A, Cannizzaro S. Dietary calcium intake in patients with inflammatory bowel disease. J Crohns Colitis. 2014; 8(4):312-317.

[PubMed: 24090907] 
What is known

- The Specific Carbohydrate diet is an exclusion diet used as a therapy in Inflammatory Bowel Disease.

- The SCD excludes grains, sweeteners except for honey and milk/milk products except for hard cheeses and yogurt fermented for greater than 24 hours.

\section{What is new}

- The SCD can be a nutritionally complete diet with appropriate intake of macro and micronutrients.

- Vitamin D supplementation should be evaluated for all patients on the SCD. 


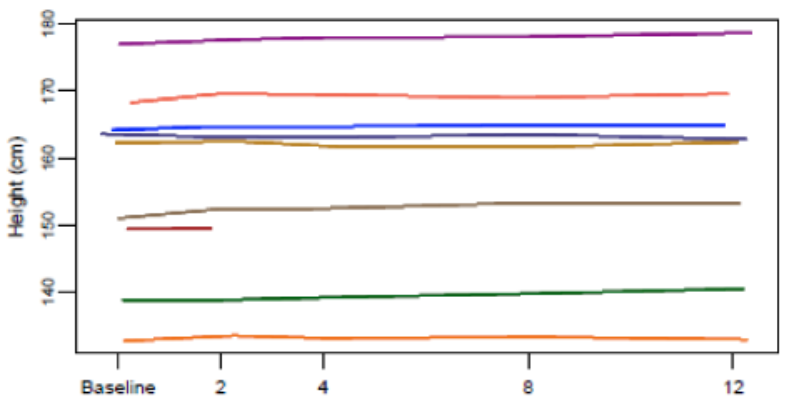

Time (Weeks)

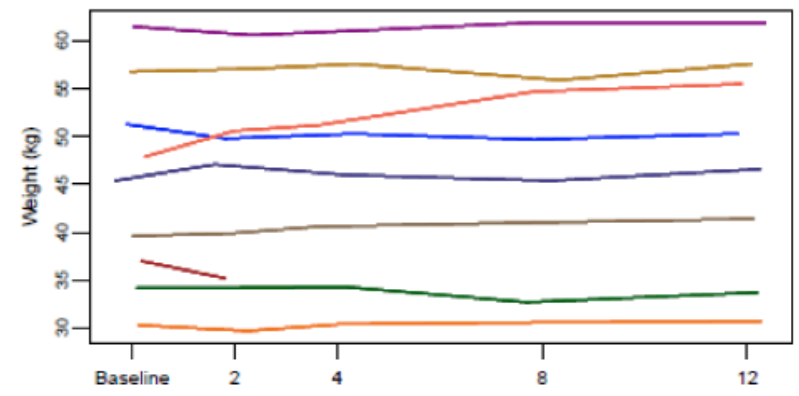

Time (Weeks)

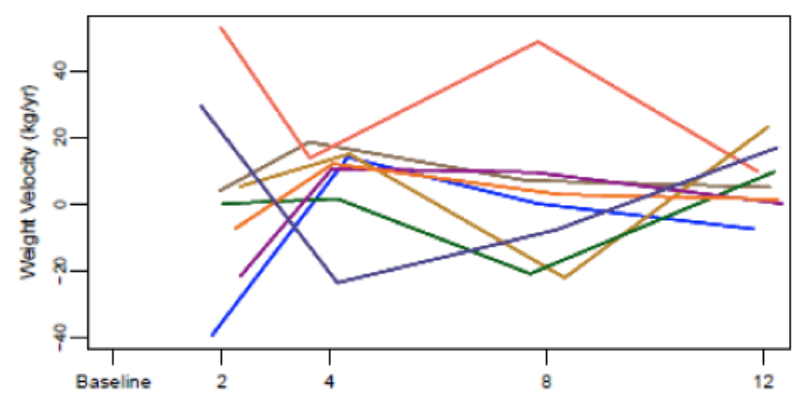

Time (Weeks)

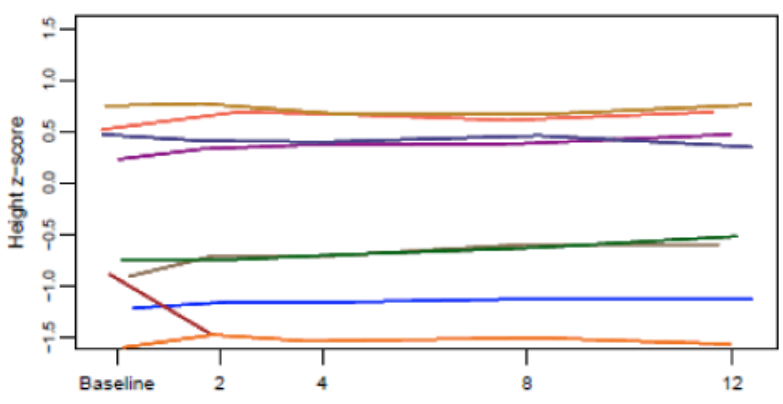

Time (Weeks)

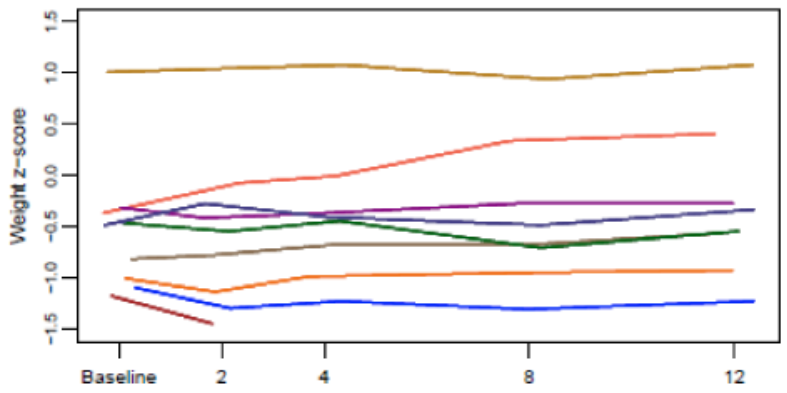

Time (Weeks)

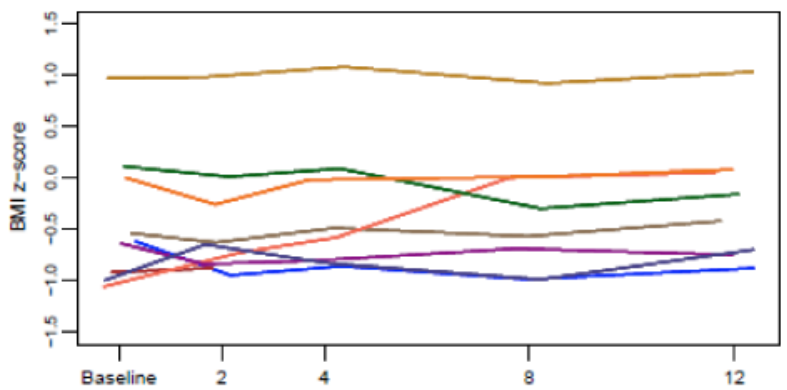

Time (Weeks)

Figure 1.

Height, weight, weight velocity, and BMI z-score for Specific Carbohydrate Diet (SCD) patients. Each color shows the profile of an individual patient. 


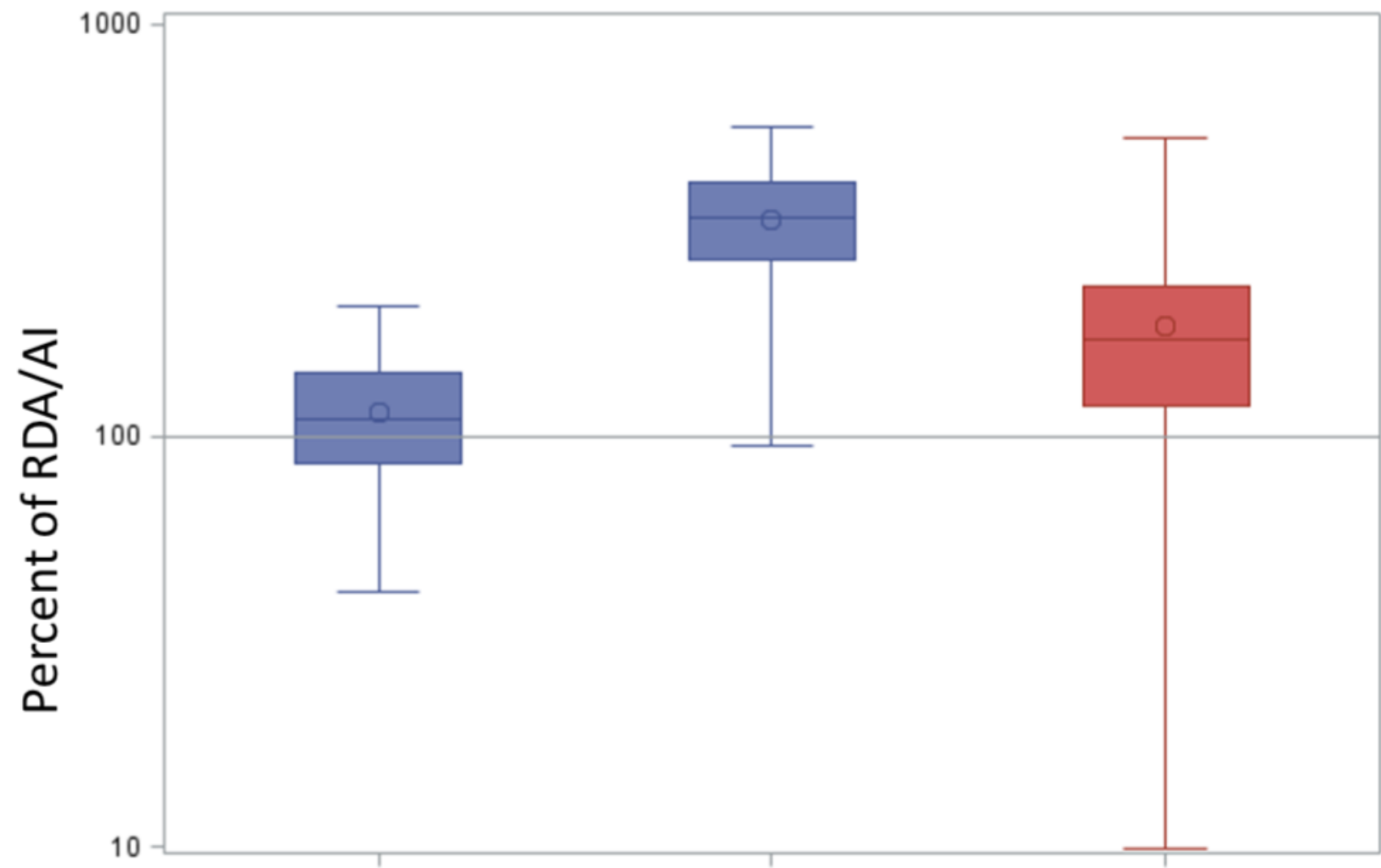

Energy

Protein

Figure 2.

Percent of Recommended Daily Allowance (RDA)/Adequate Intake (AI) for energy and protein. Specific Carbohydrate Diet (SCD) patient data are displayed in blue. National Youth Fitness Survey (NYFS) data are displayed in red. Circles shown on boxplots are means. 


\section{Vitamins}

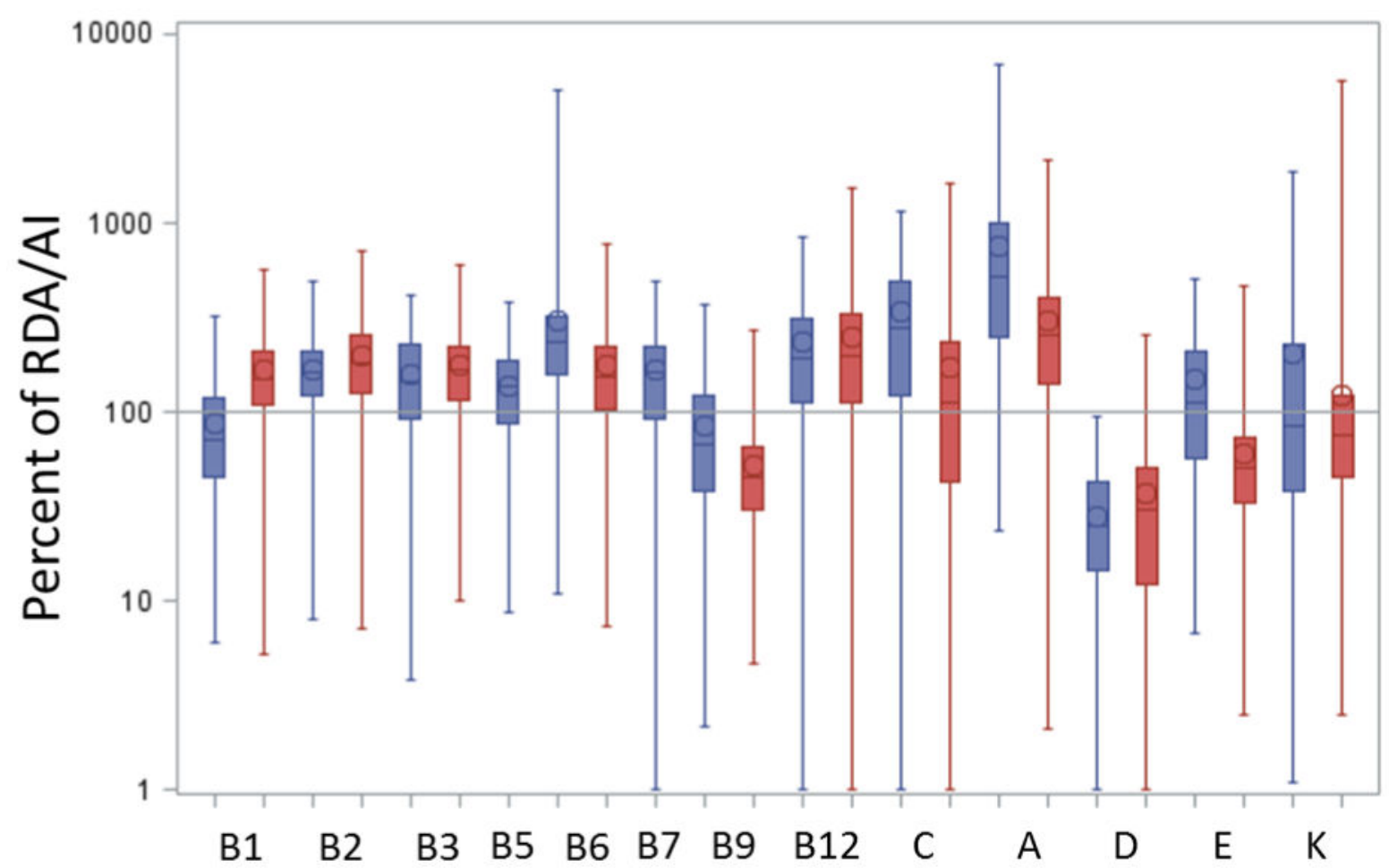

Figure 3.

Percent of Recommended Daily Allowance (RDA)/Adequate Intake (AI) for vitamins. Specific Carbohydrate Diet (SCD) patient data are displayed in blue. National Youth Fitness Survey (NYFS) data are displayed in red. Circles shown on boxplots are means. 


\section{Minerals and Trace Elements}

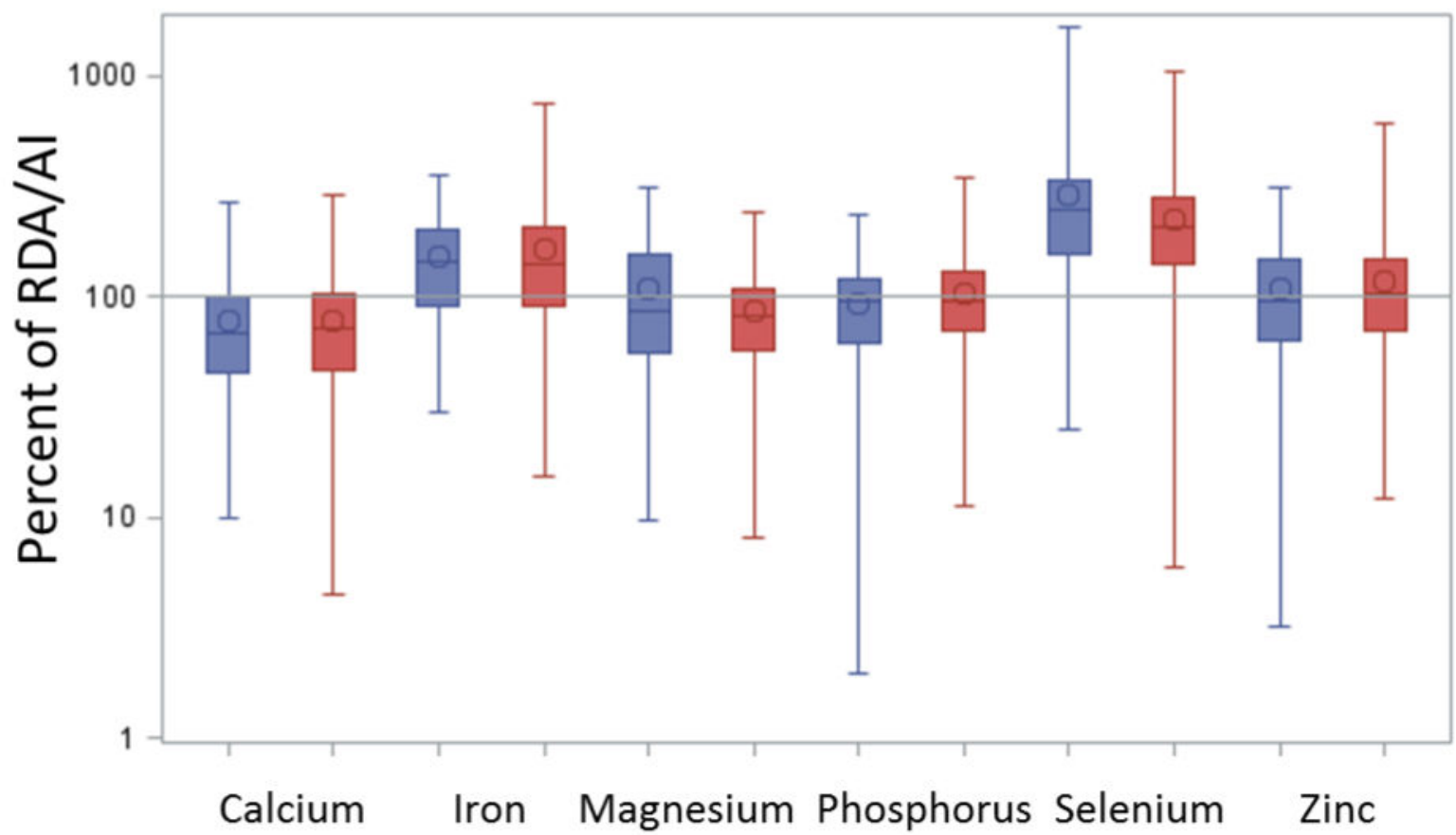

Figure 4.

Percent of Recommended Daily Allowance (RDA)/Adequate Intake (AI) for minerals and trace elements. Specific Carbohydrate Diet (SCD) patient data are displayed in blue.

National Youth Fitness Survey (NYFS) data are displayed in red. Circles shown on boxplots are means. 


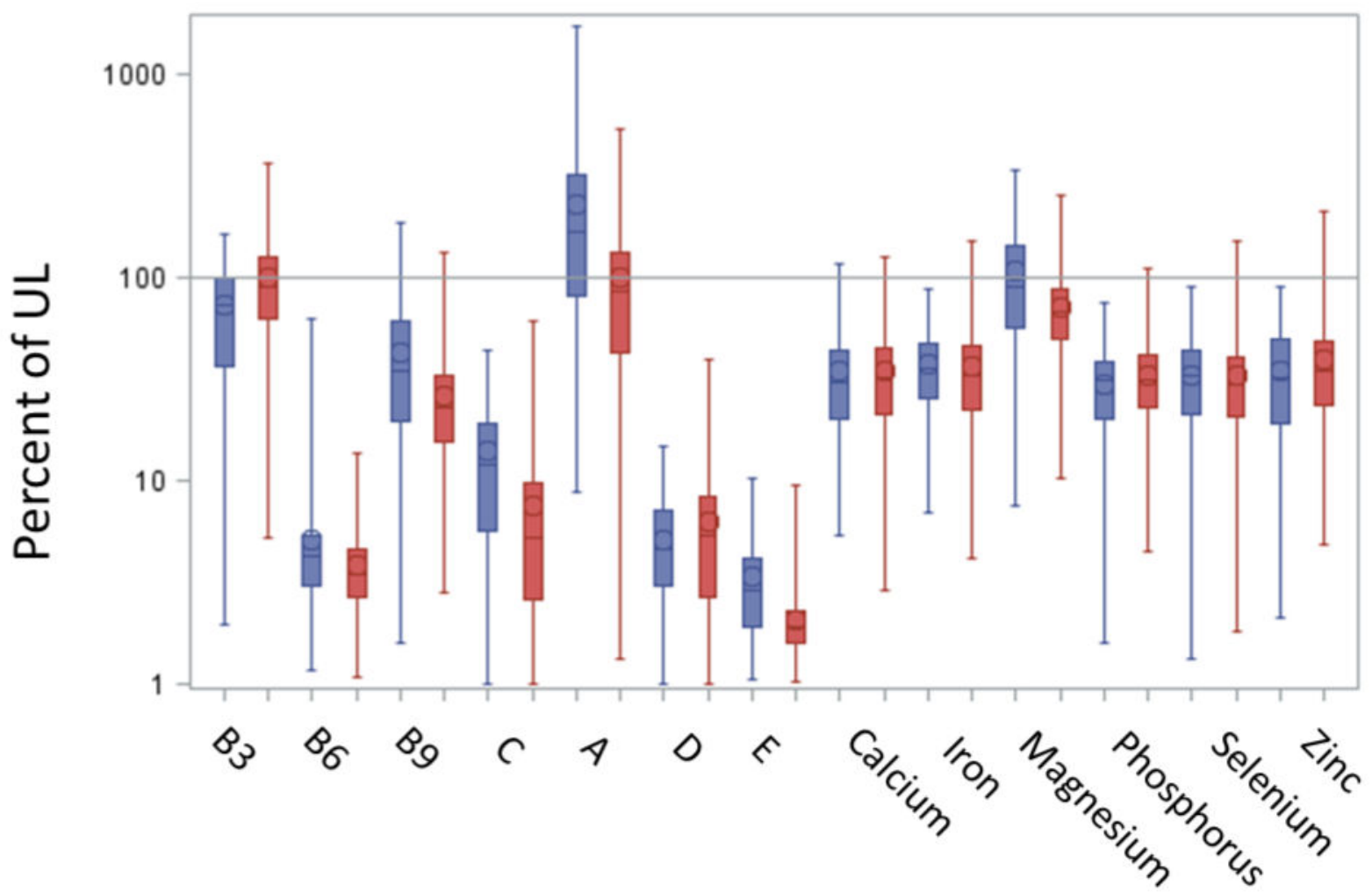

Figure 5.

Percent of Upper Limit (UL) for vitamins, minerals, and trace elements. Specific Carbohydrate Diet (SCD) patient data are displayed in blue. National Youth Fitness Survey (NYFS) data are displayed in red. Circles shown on boxplots are means. 
Table 1

Percent of Specific Carbohydrate Diet (SCD) patients and National Youth Fitness

Survey ${ }^{1}$ (NYFS) participants achieving nutritional adequacy

\begin{tabular}{|c|c|c|}
\hline \multirow[t]{2}{*}{ Nutrient } & \multicolumn{2}{|c|}{ Percent Achieving Nutritional Adequacy } \\
\hline & SCD Patients $(\mathrm{N}=8)$ & NYFS Patients $(\mathrm{N}=605)$ \\
\hline \multicolumn{3}{|l|}{ Vitamins } \\
\hline B1 & 37.5 & 79.2 \\
\hline B2 & 87.5 & 83.6 \\
\hline B3 & 87.5 & 81.0 \\
\hline B5 & 75.0 & -- \\
\hline B6 & 87.5 & 77.2 \\
\hline B7 & 75.0 & -- \\
\hline B9 & 37.5 & 6.1 \\
\hline B12 & 87.5 & 79.3 \\
\hline $\mathrm{C}$ & 100 & 52.9 \\
\hline A & 100 & 83.6 \\
\hline $\mathrm{D}$ & 0 & 5.1 \\
\hline E & 75.0 & 12.2 \\
\hline K & 62.5 & 34.7 \\
\hline \multicolumn{3}{|c|}{ Minerals and Trace Elements } \\
\hline Calcium & 12.5 & 26.8 \\
\hline Iron & 75.0 & 70.1 \\
\hline Magnesium & 50.0 & 30.9 \\
\hline Phosphorus & 37.5 & 46.6 \\
\hline Selenium & 100 & 89.3 \\
\hline Zinc & 50.0 & 52.4 \\
\hline Energy & 62.5 & -- \\
\hline Protein & 100 & 84.3 \\
\hline
\end{tabular}


Table 2

Baseline demographic and clinical characteristics of Specific Carbohydrate Diet (SCD) patients

\begin{tabular}{|l|l|}
\hline Characteristic & Mean \pm Standard Deviation or Frequency (Percent) \\
\hline Age & $13.6 \pm 2.0$ \\
\hline Gender & \\
\hline Female & $4(44 \%)$ \\
\hline Male & $5(56 \%)$ \\
\hline Disease duration & $1.6 \pm 1.7$ \\
\hline Supplementation & \\
\hline Multivitamins & $\begin{array}{l}\text { Pt 1: 2000 IU/d D3 + MV } \\
\text { Pt 2: SCD adult MVI + 2000 IU/d D3 } \\
\text { Pt 3: Folic acid + vitamin D + Iron }+ \text { MVI } \\
\text { Pt 4: 2000 IU/d D3 } \\
\text { Pt 5: SCD vitamin D } \times 2000 \text { IU/d } \\
\text { Pt 6: non SCD MVI with minerals } \\
\text { Pt 7: 2000 IU/d D3 } \\
\text { Pt 9: 6000 IU/d D3 + fish oil } \times 1000 \mathrm{mg} / \mathrm{d}+\text { curcumin/theracumin 30 mg/d }\end{array}$ \\
\hline Probiotics & Pt 9: VSL \#3 \\
\hline & \multicolumn{2}{|l}{} \\
\hline
\end{tabular}

